\title{
Effects of feed intake on the diversity and population density of homoacetogens in the large intestine of pigs
}

\author{
Hiroki Matsui ${ }^{1, *}$, Ayumi Mimura ${ }^{1,2}$, Sakiko Maekawa ${ }^{1}$, and Tomomi Ban-Tokuda ${ }^{1}$
}

* Corresponding Author: Hiroki Matsui Tel: +81-59-231-9593, Fax: +81-59-231-9540,

E-mail: matsui@bio.mie-u.ac.jp

${ }^{1}$ Graduate School of Bioresources, Mie University, Tsu, Mie 514-8507, Japan

${ }^{2}$ Graduate School of Bioagricultural Sciences, Nagoya University, Chikusa, Nagoya 464-8601, Japan

ORCID

Hiroki Matsui

https://orcid.org/0000-0002-0122-2735

Ayumi Mimura

https://orcid.org/0000-0002-6212-7088

Sakiko Maekawa

https://orcid.org/0000-0001-6596-4706

Tomomi Ban-Tokuda

https://orcid.org/0000-0001-7688-3848

Submitted Jul 5, 2018; Revised Aug 30, 2018; Accepted Feb 12, 2019
Objective: Homoacetogens play important roles in the production of acetate in the large intestine of monogastric mammals. However, their diversity in the porcine large intestine is still unknown. Marker gene analysis was performed to assess the effects of energy level on the diversity and population densities of homoacetogens in porcine feces.

Methods: Crossbred pigs were fed high or low energy-level diets. The high-intake (HI) diet was sufficient to allow a daily gain of $1.2 \mathrm{~kg}$. The low-intake (LI) diet provided 0.6 times the amount of energy as the $\mathrm{HI}$ diet. Genetic diversity was analyzed using formyltetrahydrofolate synthetase gene (FHS) clone libraries derived from fecal DNA samples. FHS DNA copy numbers were quantified using real-time polymerase chain reaction.

Results: A wide variety of $F H S$ sequences was recovered from animals in both treatments. No differences in FHS clone libraries between the HI and LI groups were found. During the experimental period, no significant differences in the proportion of FHS copy numbers were observed between the two treatment groups.

Conclusion: This is the first reported molecular diversity analysis using specific homoacetogen marker genes from the large intestines of pigs. There was no observable effect of feed intake on acetogen diversity.

Keywords: Feed Intake; Formyltetrahydrofolate Synthetase (FTHFS) Gene (FHS); Homoacetogen; Large Intestine; Pig

\section{INTRODUCTION}

Mammalian animals harbor an enormously diverse and dense microbiota in their gastrointestinal tract (GIT), particularly in the large intestine. This mutualistic microbiota plays an important role in determining animal nutrition and health. Dietary components that have not been digested in the stomach and small intestine, primarily indigestible carbohydrates, enter the large intestine and provide fermentation substrata for gut microbes. Short-chain fatty acids (SCFAs) produced during fermentation are transported across the epithelial cells of the large intestine and utilized as energy sources by the animal [1]. Fermentation by microbes in the GIT is important for energy homeostasis [2]. In pigs, SCFAs produced in the large intestine supply up to $30 \%$ of the energy required for maintenance [3]. Thus, SCFAs in the porcine large intestine are important energy sources and can have large effects on productivity.

In the large intestine of monogastric mammals and in the rumen of ruminants, homoacetogenic bacteria (acetogens) produce acetate by reducing $\mathrm{CO}_{2}$ using $\mathrm{H}_{2}$ via a pathway known as reductive acetogenesis [4-7]. A previous in vitro experiment using ${ }^{13} \mathrm{CO}_{2}$ found that acetogens compete with methanogens for $\mathrm{H}_{2}$ in the large intestine of pigs [4]. Gases such as $\mathrm{CO}_{2}$, hydrogen, methane, and ammonia are produced by fermentation. Production 
of these gases is lower in the hind gut of monogastric animals than in the rumen of ruminants. Several studies have demonstrated that this disparity in methane production between monogastric species and ruminants is due to the increased production of propionate using hydrogen during reductive acetogenesis in monogastric species $[5,8,9]$. Thus, acetogens are vital for energy homeostasis in the large intestines of host animals. In our recent publication, the diversity of methanogens in the large intestines of pigs varied significantly in animals fed high intake (HI) diets relative to those fed low intake (LI) diets [10]. Therefore, we hypothesized that the diversity of acetogens might also differ between animals fed $\mathrm{HI}$ and LI diets.

Drake et al [11] pointed out that the development of $16 \mathrm{~S}$ ribosomal RNA gene (16S rDNA) oligonucleotide primers that exclusively target all known acetogens is impossible, as their 16S rDNA sequences are not monophyletic and are often very closely related to non-acetogen taxa. Formyltetrahydrofolate synthetase (FTHFS) is a key enzyme in acetogenesis encoded by the FHS gene. Oligonucleotide primers that amplify the partial gene sequence encoding the enzyme gene have been developed and applied in acetogen diversity analyses [12]. Acetogen diversity has been successfully assessed in the rumen [13,14], human large intestine [7], and ostrich ceca [15] using FHS gene clone libraries. However, little is known about the diversity and population density of acetogens in the porcine large intestine.

In this study, pigs were fed 2 different diets each with a different intake level. We examined the effect of intake level on acetogen diversity and population size in pig feces. The FHS clone libraries were used to assess acetogen diversity. Real-time polymerase chain reaction (PCR) targeting the FHS gene was employed to quantify acetogen population densities.

\section{MATERIALS AND METHODS}

\section{Animals and diets}

Animals were handled according to the guidelines of Mie University. Eight crossbred pigs (Camborough $\times$ Duroc; average body weight $44.5 \pm 4.5 \mathrm{~kg}$ ) were used in the study. After a 1-wk adaptation period, the animals were fed for $8 \mathrm{wks}$. The animals were individually caged during the experiment. Feed intake and body weight was recorded weekly.

The ingredients and chemical compositions of each diet are shown in Table 1. Both diets were mush-type diets. The animals were equally divided into 2 dietary groups, with each diet varying in its energy content based on differing provisioning rates. In the HI diet group, animals were fed a diet resulting in a daily gain of $1.2 \mathrm{~kg}$. The LI diet provided 0.6 times the amount of energy that satisfied daily gain of $1.2 \mathrm{~kg}$. Dietary provisioning rates were calculated based on the ani-
Table 1. Ingredients and chemical compositions of diets

\begin{tabular}{lc}
\hline Items & \\
\hline Ingredients (\%) & 69.1 \\
Maize & 10.0 \\
Wheat bran & 10.0 \\
Soybean meal & 5.0 \\
Rapeseed meal & 3.0 \\
Defatted rice bran & 1.0 \\
Fish meal & 0.15 \\
Dicalcium phosphate & 0.93 \\
Calcium carbonate & 0.02 \\
Salt & 0.02 \\
Vitamin B complex & 0.02 \\
Trace minerals & \\
Chemical composition & 3.36 \\
DE (MJ/kg) & 76.2 \\
TDN (\%) & 13.8 \\
Crude protein (\%) & 4.47 \\
Crude fiber (\%)
\end{tabular}

$\mathrm{DE}$, digestible energy; TDN, total digestible nutrients.

mals' weights and according to the Japanese Feeding Standard for Swine [16]. Animals were fed at 09:00 and 16:00 each day.

\section{Sample collection and DNA extraction}

Fecal samples were collected manually from the rectum (by gloved hands) at 09:00 on the first day of weeks 0,4 , and 8 . Fecal samples were put in plastic bags and immediately placed on ice. The samples were then transferred to the laboratory where they were stored at $-80^{\circ} \mathrm{C}$ before analysis.

Frozen fecal samples were thawed at $25^{\circ} \mathrm{C}$. Total DNA was extracted from $0.25 \mathrm{~g}$ of each fecal sample using the UltraClean Fecal DNA Isolation kit (MO BIO Laboratories, Inc., Carlsbad, CA, USA). For the construction of clone libraries, the concentrations of DNA samples from each animal were adjusted to $15 \mathrm{ng} / \mu \mathrm{L}$. The extracted DNA was stored at $-25^{\circ} \mathrm{C}$ until analysis.

\section{Construction of FHS clone libraries and diversity analyses}

DNA samples taken on week 8 were used to construct clone libraries. DNA solutions from each animal in each treatment were combined in equal parts and used as template for PCR. The FHS clone libraries were constructed following the methods of Matsui et al [14].

Sequences from the cloned DNA fragments were analyzed as described elsewhere [17]. The DNA sequences were used as search queries in BlastX [18]. Operational taxonomic units (OTUs), coverage, the Shannon-Wiener index $\left(H^{\prime}\right)$, and Chaol were calculated using the DOTUR program [19]. Sequences were assigned to individual OTUs based on a $98 \%$ amino acid sequence similarity criterion [20]. The amino acid sequences 
were aligned using ClustalX ver. 2.0 [21], and phylogenetic trees were constructed using the neighbor-joining method [22]. Bootstrapping (1,000 resamplings) was used to estimate the confidence of branch patterns. The statistical significance of differences between the clone libraries was evaluated using the webLIBSHUFF program (http://libshuff.mib.uga.edu) [23].

Forty amino acid residues within the deduced amino acid sequence of FHS were used to calculate the homoacetogen similarity (HS) scores for FHS sequences. Sequences were then extracted from the alignment file and calculated using the methods described by Henderson et al [24]. Sequences with high HS scores $(\geq 80 \%)$ were identified as possible homoacetogens.

\section{Real-time polymerase chain reaction assays}

The DNA copy number of FHS and the DNA copy number of $16 \mathrm{~S}$ ribosomal RNA ( $16 \mathrm{~S} \mathrm{rDNA}$ ) of the total bacteria found in fecal samples were quantified using real-time PCR. All real-time PCR assays were performed using an ABI Prism 7000 Sequence Detection System (Applied Biosystems, Foster City, CA, USA) and SYBR green PCR master mix (Applied Biosystems, USA). The reaction mixture $(25 \mu \mathrm{L})$ consisted of $1 \mu \mathrm{L}$ of template, $12.5 \mu \mathrm{L}$ of SYBR green master mix, and sterilized Milli-Q water. A primer pair, FTHFS for (5'-TTYAC WGGHGAYTTCCATGC-3') and FTHFS rev (5'-GTATT GDGTYTTRGCCATACA-3') was used in FHS analysis [25]. Assays were carried out using the following cycle conditions: 1 cycle at $50^{\circ} \mathrm{C}$ for $2 \mathrm{~min}, 1$ cycle at $95^{\circ} \mathrm{C}$ for $2 \mathrm{~min}$ to allow initial denaturation, 40 cycles at $95^{\circ} \mathrm{C}$ for $15 \mathrm{~s}$, and $60^{\circ} \mathrm{C}$ for $1 \mathrm{~min}$ to allow primer annealing and product elongation. Amplicon specificity was determined via dissociation curve analysis of PCR end products by increasing the temperature at a rate of $1^{\circ} \mathrm{C} / 30 \mathrm{~s}$ from $60^{\circ} \mathrm{C}$ to $95^{\circ} \mathrm{C}$. For $F H S$, standard DNA was prepared using an $\mathrm{FHS}$ gene fragment from Blautia producta.

Quantification of 16S rDNA was performed following the methods of Guo et al [26]. A primer pair, 530f (5'-GTGCCAG CMGCCGCGG-3') and 920r (5'-GTCAATTCCTTTGAG TTT-3') was used in rDNA assays. Standard DNA was prepared using a $16 \mathrm{~S}$ rDNA fragment from Escherichia coli. The number of copies of FHS was expressed as the number of copies relative to the total number of bacteria.

\section{Nucleotide sequence accession numbers}

All nucleic acid sequences obtained in this study were deposited in the DNA Data Bank of Japan, European Molecular Biology Laboratory, and GenBank databases under accession numbers AB623795-AB623894.

\section{Statistical analysis}

Differences in the measured parameters between treatments were compared using unpaired $t$-test. Differences in mean measured parameters between weeks were compared using paired t-test. All statistical analyses were performed using a commercially available computer program (StatView; SAS Institute Inc., Cary, NC, USA). Differences were considered significant at $\mathrm{p}<0.05$.

\section{RESULTS}

\section{FHS gene diversity in porcine fecal material}

The nucleotide sequence of the cloned DNA fragment was 1,095 to $1,104 \mathrm{bp}$ in length. The deduced amino acid residues were 365 to $370 \mathrm{bp}$ in length.

A total of 50 clones were randomly selected and analyzed for each library. Clones in the FHS library identified based on the analysis of material from the HI group were classified into 17 OTUs, with 20 OTUs being classified based on material from the LI group. The coverage of the FHS libraries derived from samples in the HI treatment and the LI treatment were $84 \%$ and $82 \%$, respectively. The $H^{\prime}$ of the FHS libraries derived from samples from the HI and LI treatments were 2.532 and 2.749 , respectively. The Chao- 1 estimation of HI and LI treatment were 31 and 27 species, respectively. No statistical differences were detected between the FHS clone libraries derived from the HI and LI treatments (data not shown).

A phylogenetic tree was constructed using the deduced FHS amino acid sequences recovered in our study and FHS sequences in the databases (Figure 1). The OTUs were classified into 5 clades according to their phylogenetic positioning.

Table 2 summarizes the sequence similarities of OTUs relative to the FHS sequences deposited in the public databases and the HS scores calculated by Henderson et al [24]. Clade I consists of 5 OTUs ( 12 clones) isolated from animals in the HI group and 7 OTUs (14 clones) isolated from animals in the LI group (Table 2). Five OTUs (H45 and H47 and L20, L54, and L66) showed high similarity to the FHS sequence of Ruminococcusceaea bacterium D16; however, L54 showed lower similarity and was, thus, likely only distantly related to the FHS of Ruminococcusceaea bacterium D16. L41 showed the highest similarity and was closely related to the ostrich gut clone OstMCfhs-11. H44, H61, L32, and L44 also exhibited high similarities to OstMCfhs-11. Three OTUs (7 clones) isolated from animals in the HI treatment and 6 OTUs (13 clones) isolated from animals in the LI treatment were classified into clade II. L34 showed high similarity to the authentic acetogen, Marvinbryantia formatexigens (97\%). H42 and L71 also exhibited high similarities to the authentic acetogen, Bryantella hydrogenotrophica (97\% and 96\%). H44 and L15 exhibited high similarities to the ostrich gut clones OstPCfhs-36 (99\%) and OstMCfhs-22 (98\%). The rest of the OTUs (H43, L09, L42, and L84) were the most similar to the ostrich clone 


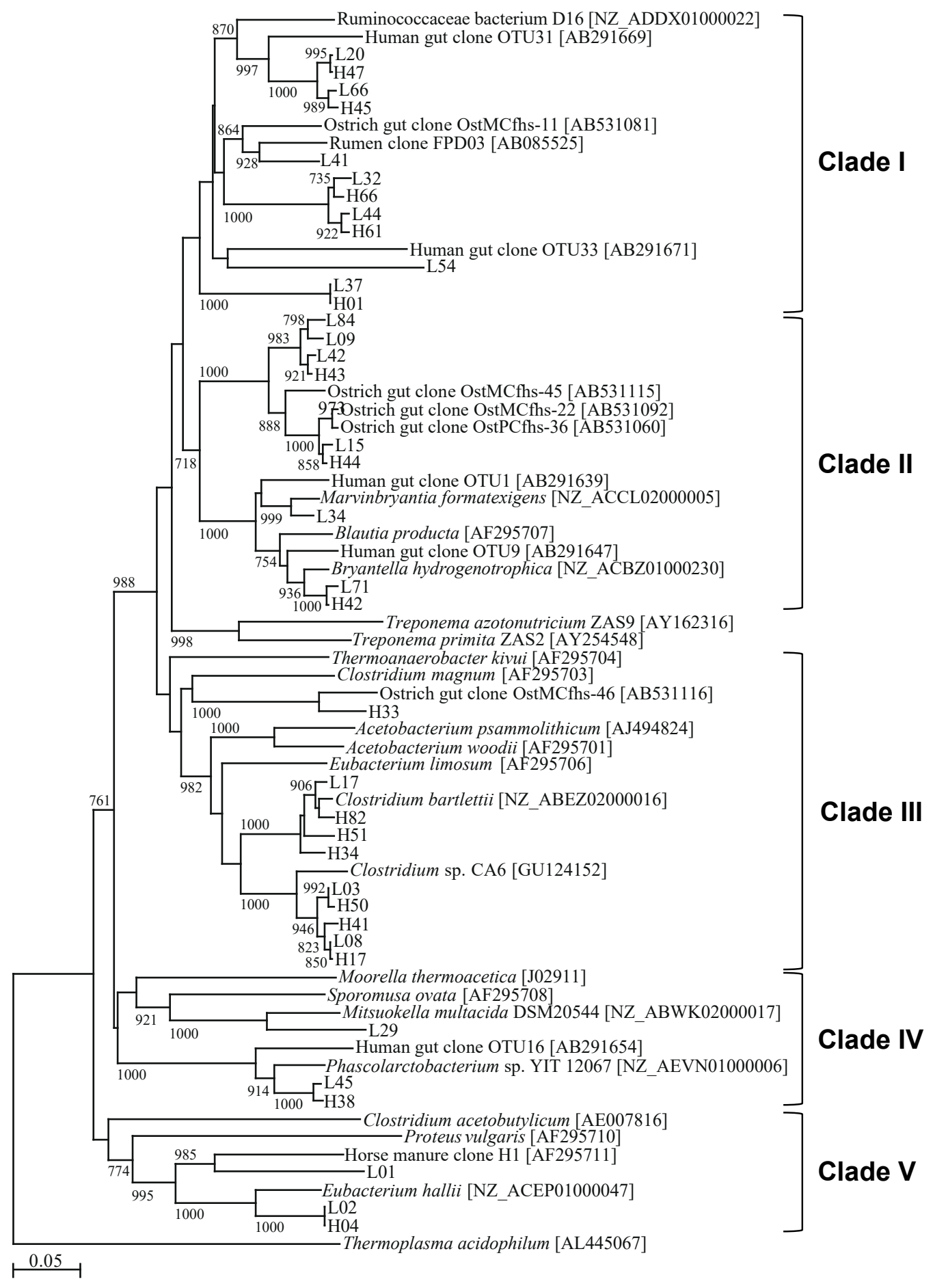

Figure 1. Phylogenetic tree based on formyltetrahydrofolate synthetase (FHS) sequences recovered from pig feces. The accession number of each sequence is shown in brackets. The scale bar represents 0.05 substitutions per amino acid position. Bootstrap values based on 1,000 trees are shown at each node; only values $\geq 700$ are shown. Sequence names are the representative clone name for each operational taxonomic unit.

OstMCfhs-45. Clade III consisted of 7 OTUs isolated from animals in the HI group and 3 from animals in the LI group. Twenty-three clones were isolated from animals in the HI group, almost double the number of clones isolated from animals in the LI group. H33 exhibited similarity to the os- trich gut clone OstMCfhs-46. Three OTUs, H34, H51, and H82 (5 clones) isolated from animals in the HI group and 1 OTU, L17 (1 clone), isolated from animals in the LI group showed high similarity to FHS from Clostridium bartlettii (96\% to 98\%). Three OTUs, H17, H41, and H50 (15 clones) 
Table 2. Blast search results and homoacetogen similarity (HS) score of amino acid sequences and operational taxonomic units (OTU) derived from 2 FHS clone libraries derived from pig fecal material

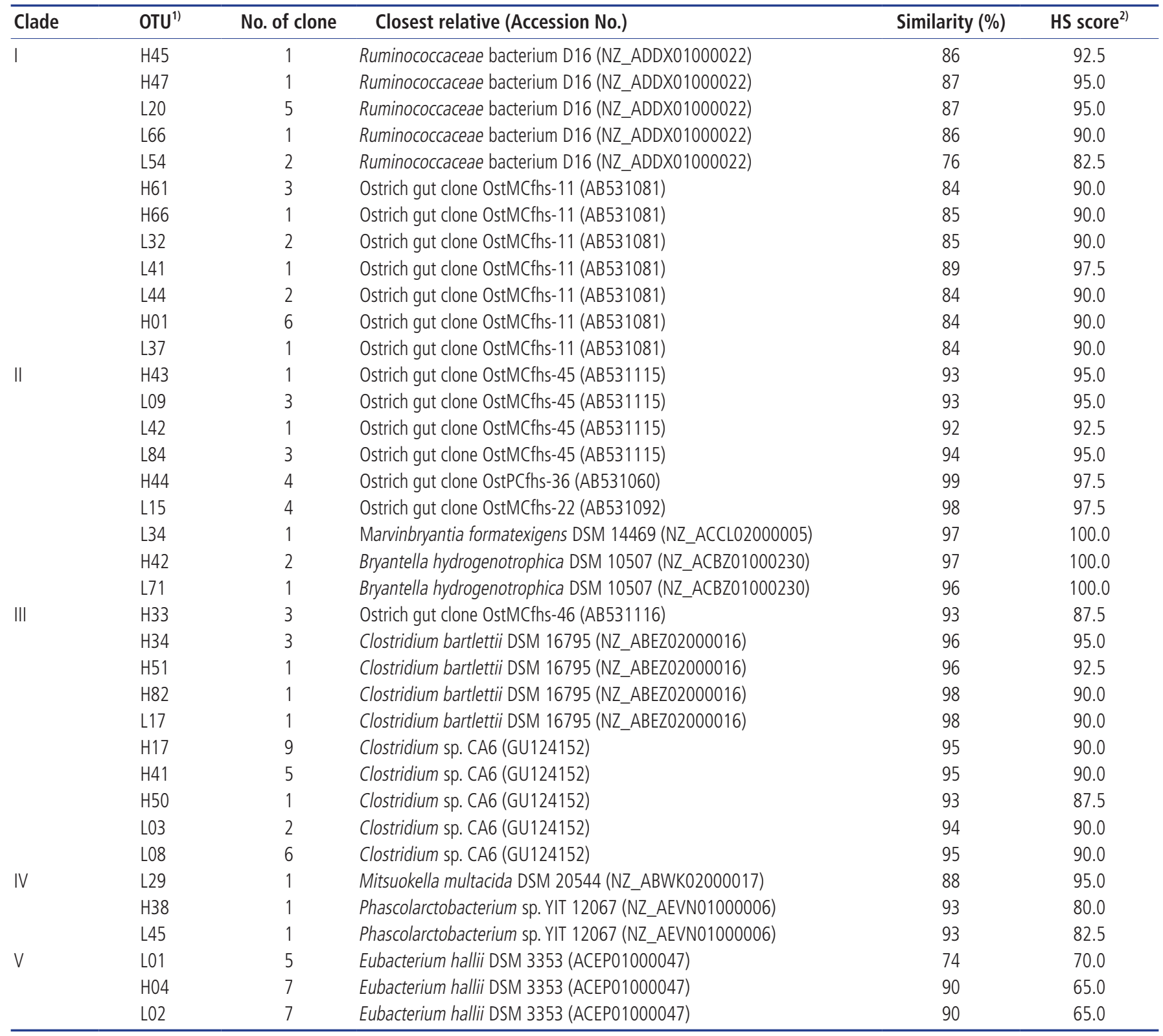

1) OTU names starting with " $\mathrm{H}$ " and "L" were derived from the high-intake library and a low-intake library, respectively. OTU names are representative of the clone name of each OTU.

${ }^{2)} \mathrm{HS}$ score was calculated according to the methods of Henderson et al [24].

isolated from animals in the HI group and 2 OTUs, L03 and L08 (8 clones), isolated from animals in the LI group exhibited relatively high similarities to Clostridium sp. CA6 (93\% to 95\%). Both clade IV and V contained only 3 OTUs. L29 from clade IV showed similarity to the FHS sequence of Mitsuokella multacida. Two other OTUs showed similarity to the FHS sequence of Phascolarctobacterium sp. YIT 12067. In clade V, all OTUs showed similarity to the FHS sequence of Eubacterium hallii. The HS scores of all OTUs in this clade were lower than 80\%. L01, L02, and H04 contained 5, 7 (a total of 12), and 7 clones, respectively. Therefore, $(12 / 50) \times 100=$
$24 \%$ of clones isolated from animals in the LI group and $(7 / 50) \times 100=14 \%$ of clones isolated from animals in the HI group were not authentic acetogens.

\section{Real-time polymerase chain reaction assays}

The proportion of FHS against $16 \mathrm{~S}$ rDNA copy number was very low (Figure 2). The proportion of FHS in the feces of animals in the $\mathrm{HI}$ group increased at wk 4 but had significantly decreased by the 8 th wk $(\mathrm{p}<0.05)$. The proportion of FHS in the feces of animals in the LI group continuously decreased from the start to the end of the experiment. The proportion 


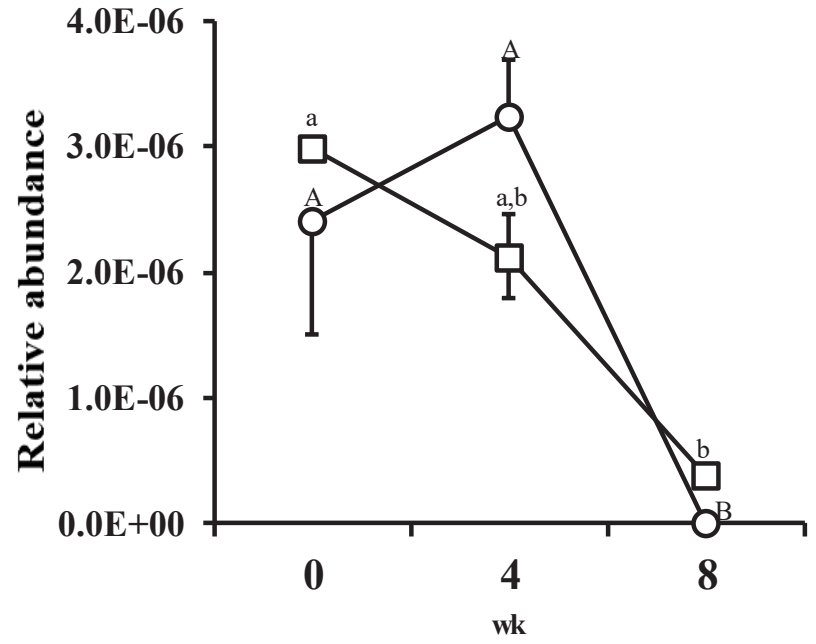

Figure 2. Changes in the relative abundance of formyltetrahydrofolate synthetase (FHS) genes against 16S ribosomal RNA genes from all bacteria recovered from porcine feces over the course of the experiment. Figure legend: $O$, high-intake diet; $\square$, low-intake diet. Error bar shows standard error $(n=4)$. ${ }^{A, B}$ Letters indicate significant differences between mean values for each week in the high-intake treatment $(p<0.05)$. ${ }^{a, b}$ Letters indicate significant differences between mean values for each week in the low-intake treatment $(p<0.05)$.

of FHS at the 8th week was significantly lower than the proportion at wk 0 and wk $4(\mathrm{p}<0.05)$. During the experimental period, no significant differences in the proportion of FHS between treatments were observed.

\section{DISCUSSION}

To date, FHS clone library analyses of the bovine rumen [13, 24], human large intestine [7], Tammar wallaby forestomach [13], and ostrich ceca [15], have been performed. However, there is no existing report on FHS gene diversity in the large intestine of pigs. This is, thus, the first reported molecular diversity analysis of homoacetogens in the pig large intestine.

A wide variety of $F H S$ gene fragments were recovered from pig feces. Large numbers of FHS clones from unknown bacteria were recovered from the bovine rumen [14,24], human large intestine [7], Tammar wallaby forestomach [13], and ostrich ceca [15]. Similarly, the libraries derived from pig fecal samples in this study also contained a number of FHS sequences from unknown bacteria. Therefore, the GIT is probably colonized by many uncultivated acetogens. In the large intestines of pigs, methanogen diversities in the $\mathrm{HI}$ and LI groups was significantly different [10]. Unexpectedly, however, feed intake level did not affect acetogen diversity. This suggests that acetogen diversity in the large intestines of pigs was not related to host animal energy homeostasis.

The FHS clone libraries recovered in this study may contain FHS sequences from non-acetogenic bacteria [13,14]. The isolation and characterization of acetogenic bacteria is, thus, required to fully understand the diversity and function of acetogens in the pig large intestine.

The proportion of $F H S$ decreased as pigs gained weight in both treatments (Figure 2). The exact reason for this is unclear, but the results suggest that the internal environment of the large intestine became increasingly hostile to acetogens as the animals gained weight. The proportion of FHS was also very low, and energy level had no significant effect on the proportion of FHS, suggesting that acetogen population density was also not affected by host energy homeostasis.

Finally, the same primer set was used in both the quantification of FHS and in diversity analyses. According to the results of the FHS diversity analysis, $24 \%$ of all amplified FHS fragments of in LI and 14\% in HI, not all FHS fragments amplified with primer set used in this study were authentic acetogens. Therefore, the values obtained from the real-time PCR may have been somewhat overestimated.

Further study is required to clarify the reason the reduction of population density of acetogens during fattening period.

\section{CONFLICT OF INTEREST}

We certify that there is no conflict of interest with any financial organization regarding the material discussed in the manuscript.

\section{ACKNOWLEDGMENTS}

The authors thank Professor Kazuo Sakka (Graduate School of Mie University, Japan) for his instruction on real-time PCR. Nucleotide sequencing was performed at the Life Science Research Center (Center for Molecular Biology and Genetics), Mie University (Tsu, Japan).

\section{REFERENCES}

1. Engelhardt WV. Absorption of short-chain fatty acids from the large intestine. In: Cummings JH, Rombeau JL, Sakata T, editors. Physiological and clinical aspects of short-chain fatty acids. Cambridge, UK: Cambridge University Press; 1995. p. 149-70.

2. Arora T, Sharma R. Fermentation potential of the gut microbiome: implications for energy homeostasis and weight management. Nutr Rev 2011;69:99-106. https://doi.org/10.1111/ j.1753-4887.2010.00365.x

3. Rose CJ, Hume ID, Farrell DJ. Fibre digestion and volatile fatty acid production in domestic and feral pigs. In: Farrell DJ, editor. Recent advances in animal nutrition in Australia. Armidale, Australia: University of New England Press; 1987. p. 347-60.

4. De Graeve KG, Grivet JP, Durand M, et al. Competition 
between reductive acetogenesis and methanogenesis in the pig large-intestinal flora. J Appl Bacteriol 1994;76:55-61.

5. Lajoie SF, Bank S, Miller TL, Wolin MJ. Acetate production from hydrogen and $\left({ }^{13} \mathrm{C}\right)$ carbon dioxide by the microflora of human feces. Appl Environ Microbiol 1988;54:2723-7.

6. Morvan B, Bonnemoy F, Fonty G, Gouet P. Quantitative determination of $\mathrm{H}_{2}$-utilizing acetogenic bacteria and sulfatereducing bacteria and methanogenic archaea from digestive tract of different mammals. Curr Microbiol 1996;32:129-33. https://doi.org/10.1007/s002849900023

7. Ohashi Y, Igarashi T, Kumazawa F, Fujisawa T. Analysis of acetogenetic bacteria in human feces with formyltetrahydrofolate synthetase sequences. Biosci Microflora 2007; 26:37-40. https://doi.org/10.12938/bifidus.26.37

8. De Graeve K, Demeyer DI. Rumen and hindgut fermentation: differences for possible exploitation? Mededelingen van de Faculteit Landbouwwetenschappen Rijksuniversiteit Gent; Belgium. 1988;53:1805-9.

9. Prins RA, Lankhorst A. Synthesis of acetate from $\mathrm{CO}_{2}$ in the cecum of some rodents. FEMS Microbiol Lett 1977;1:255-8.

10. Matsui H, Ishimoto-Tsuchiya T, Maekawa S, Ban-Tokuda T. Diversity and population density of methanogens in the large intestine of pigs fed diets of different energy levels. Anim Sci J 2018:89:1468-74. https://doi.org/10.1111/asj.13083

11.Drake HL, Küsel K, Matthies C. Ecological consequences of the phylogenetic and physiological diversities of acetogen. Antonie Van Leeuwenhoek 2002;81:203-13. https:/doi.org/ 10.1023/A:1020514617738

12. Lovell CR, Leaphart AB. Community-level analysis: key genes of $\mathrm{CO}_{2}$-reductive acetogenesis. Methods Enzymol 2005;397: 454-69. https://doi.org/10.1016/S0076-6879(05)97028-6

13. Gagen EJ, Denman SE, Padmanabha J, et al. Functional gene analysis suggests different acetogen populations in the bovine rumen and Tammar wallaby forestomach. Appl Environ Microbiol 2010;76:7785-95. https://doi.org/10.1128/AEM. 01679-10

14. Matsui H, Kojima N, Tajima K. Diversity of the formyltetrahydrofolate synthetase gene (fhs), a key enzyme for reductive acetogenesis, in the bovine rumen. Biosci Biotechnol Biochem 2008;72:3273-6. https://doi.org/10.1271/bbb.70375

15. Matsui H, Yoneda S, Ban-Tokuda T, Wakita M. Diversity of the formyltetrahydrofolate synthetase (FTHFS) gene in the proximal and mid ostrich colon. Curr Microbiol 2011;62:16. https://doi.org/10.1007/s00284-010-9661-y
16. National Agriculture and Bio-oriented Research Organization. Japanese feeding standard for swine. Tokyo, Japan: Japan Livestock Industry Association; 2005.

17. Hattori K, Matsui H. Diversity of fumarate reducing bacteria in the bovine rumen revealed by culture dependent and independent approaches. Anaerobe 2008;14:87-93. https://doi. org/10.1016/j.anaerobe.2007.12.002

18. Altschul SF, Madden TL, Schäffer AA, et al. Gapped BLAST and PSI-BLAST: a new generation of protein database search programs. Nucleic Acids Res 1997;25:3389-402. https://doi. org/10.1093/nar/25.17.3389

19.Schloss PD, Handelsman J. Introducing DOTUR, a computer program for defining operational taxonomic units and estimating species richness. Appl Environ Microbiol 2005;71: 1501-6. https://doi.org/10.1128/AEM.71.3.1501-1506.2005

20. Juottonen $H$, Galand PE, Yrjälä K. Detection of methanogenic Archaea in peat: comparison of PCR primers targeting the mcrA gene. Res Microbiol 2006;157:914-21. https://doi.org/ 10.1016/j.resmic.2006.08.006

21.Larkin MA, Blackshields G, Brown NP, et al. Clustal W and Clustal X version 2.0. Bioinformatics 2007;23:2947-8. https:// doi.org/10.1093/bioinformatics/btm404

22. Saitou N, Nei M. The neighbor-joining method: A new method for reconstructing phylogenetic trees. Mol Biol Evol 1987;4: 406-25. https://doi.org/10.1093/oxfordjournals.molbev. a040454

23. Singleton DR, Furlong MA, Rathbun SL, Whitman WB. Quantitative comparisons of $16 \mathrm{~S}$ rRNA gene sequence libraries from environmental samples. Appl Environ Microbiol 2001; 67:4374-6. https://doi.org/10.1128/AEM.67.9.4374-4376.2001

24. Henderson G, Naylor GE, Leahy SC, Janssen PH. Presence of novel, potentially homoacetogenic bacteria in the rumen as determined by analysis of formyltetrahydrofolate synthetase sequences from ruminants. Appl Environ Microbiol 2010; 76:2058-66. https://doi.org/10.1128/AEM.02580-09

25. Leaphart AB, Lovell CR. Recovery and analysis of formyltetrahydrofolate synthetase gene sequences from natural populations of acetogenic bacteria. Appl Environ Microbiol 2001;67:13925. https://doi.org/10.1128/AEM.67.3.1392-1395.2001

26. Guo X, Xia X, Tang R, Zhou J, Zhao H, Wang K. Development of a real-time PCR method for Firmicutes and Bacteroidetes in faeces and its application to quantify intestinal population of obese and lean pigs. Lett Appl Microbiol 2008;47:367-73. https://doi.org/10.1111/j.1472-765X.2008.02408.x 